\title{
La naissance de la formule « LGBT » en France et en Italie : une analyse comparative des discours de mobilisation
}

The origins of the "LGBT" formula in France and in Italy: a comparative discursive analysis of collective actions

\section{Massimo Prearo}

\section{(2) OpenEdition Journals}

\section{Édition électronique}

URL : http://journals.openedition.org/conflits/18956

DOI : 10.4000/conflits. 18956

ISSN : $1777-5345$

\section{Éditeur :}

CECLS - Centre d'études sur les conflits - Liberté et sécurité, L'Harmattan

\section{Édition imprimée}

Date de publication : 30 juillet 2015

Pagination : 77-95

ISBN : 979-2-343-07175-6

ISSN : 1157-996X

\section{Référence électronique}

Massimo Prearo, «La naissance de la formule «LGBT » en France et en Italie : une analyse comparative des discours de mobilisation », Cultures \& Conflits [En ligne], 97 | printemps 2015, mis en ligne le 30 juillet 2016, consulté le 07 avril 2021. URL : http://journals.openedition.org/conflits/18956 ; DOI : https://doi.org/10.4000/conflits. 18956 


\section{La naissance de la formule "LGBT» en France et en Italie : une analyse comparative des discours de mobilisation}

\section{Massimo PREARO}

Massimo Prearo est chercheur associé (Marie Curie Fellow) au Centre de recherche Politesse / Politiques et théories de la sexualité, où il conduit des recherches sur les mouvements LGBT italiens. Il est docteur en Études Politiques de l'EHESS, a récemment publié Le moment politique de l'homosexualité. Mouvements, identités et communautés en France, PUL, 2014 et dirige actuellement un ouvrage sur Politiche dell'orgoglio. Sessualità, soggettività e movimenti sociali à parâ̂tre en 2015 (ETS, Pise). Il a traduit de l'italien pour les éditions EPEL l'ouvrage de Mario Mieli, Éléments de critiques homosexuelle. Italie : les années de plomb (2008), et a collaboré à la traduction de l'anglais de l'ouvrage d'Ann Laura Stoler, La chair de l'empire. Savoirs intimes et pouvoirs raciaux en régime colonial, traduit par Sébastien Roux, La Découverte, 2013. Il est également directeur de la revue à comité de lecture Genre, sexualité \& société.

T e point de convergence inter-associative internationale qui contribue à la Lnaissance de la «formule » LGBT est sans aucun doute la World Pride 2000, à laquelle, selon les organisateurs, ont participé plus de 500000 personnes ${ }^{1}$ :

«La World Pride n'est pas le premier événement qui associe l'aspect festif et l'aspect politique, ce n'est pas non plus la première fois qu'à Rome on marche dans la rue pour les droits, la solidarité et la visibilité. Mais c'est la première manifestation qui a pour ambition de réunir le monde entier pour revendiquer nos droits humains et civiques, en invitant les leaders et les gens provenant de tous les continents pour travailler ensemble et pour atteindre ces objectifs durant 8 jours, à l'aube du nouveau millénaire, dans le centre d'une

1. Bolognini S., «WorldPride Roma 2000, cronaca di una grande vittoria », 1er septembre 2000. Consulté le 20 avril 2015. URL: http://www.stefanobolognini.it/493/worldpride-roma2000-cronaca-di-una-grande-vittoria 
ville pleine d'histoire, patrie de gens de lettre et de penseurs, de politiques et d'athlètes, pour célébrer toutes les réalités de la communauté $G / L / B / T$. » 2

À partir d'une resignification d'anciens répertoires d'action collective, comme la Gay Pride, le référentiel LGBT a introduit un nouveau discours militant fondé sur un principe d'intégration de toutes les composantes du mouvement. Sans prétendre restituer de manière exhaustive les dynamiques complexes déployées dans les dernières décennies par les mouvements LGBT $^{3}$ dans cet effort permanent de resignification des stratégies d'action collective, je propose dans cet article une analyse ${ }^{4}$ du moment d'émergence de la formule «LGBT » comme référentiel ayant contribué à la stabilisation d'un espace du militantisme $\mathrm{LGBT}^{5}$ et à une redistribution des positions relatives des organisations en jeu dans cet espace ${ }^{6}$.

L'acronyme LGBT apparait à l'occasion de différentes réunions de préparation de la World Pride 2000 à Rome, au moment où commence à s'affirmer l'usage associatif et militant du référentiel LGBT, déjà utilisé en contexte anglophone. L'idée d'organiser en 2000 une grande manifestation à Rome fut lancée dès 1995 par le président de l'association italienne Arcigay, Franco Grillini. Lors de la réunion de l'European Pride Organiser Association (EPOA) en 1996, la proposition d'attribuer à la ville de Rome le titre d'Europride 2000 fut discutée et accueillie favorablement, puis présentée à la Conférence annuelle en septembre 1996 à Paris, et ratifiée ensuite lors de l'Assemblée annuelle de l'EPOA en 1997 à Stockholm. Mais les conflits autour de l'organisation des Gay Prides Nationales italiennes contribuèrent à rendre le climat des réunions difficiles. Le Circolo di Cultura Omosessuale Mario Mieli de Rome décide alors de se rapprocher de l'International

2. Melvin D.O., «Sei mesi al World Pride », Aut, 3/12, 2000, p. 24. Je souligne.

3. Sur les transformations récentes des mouvements LGBT voir Johnson C., Paternotte D., Tremblay M. (eds.), The Lesbian and Gay Movement and the State. Comparative Insights into a Transformed Relationship, Farnham, Ashgate, 2011 ; ainsi que Ayoub P., Paternotte D., The Ashgate Research Companion to Lesbian and Gay Activism, Farnham, Ashgate, 2015.

4. Les analyses avancées ici sont le résultat d'une recherche en cours sur les mouvements LGBT italiens. Elles sont basées sur des recherches dans les archives du Centre de Documentation Homosexuel «Il Cassero » de Bologne, sur une première période d'observation et sur une première série d'entretiens réalisées au sein du monde associatif et militant LGBT. Pour le cas français, les analyses s'appuient sur les recherches doctorales conduites dans le cadre d'une thèse en Études politiques de l'EHESS (CESPRA) «La genèse des mouvements d'affirmation identitaire : homosexualité et identité à l'âge de l'autonomie ", 2011. Dans la mesure où il s'agit d'une recherche en cours, et par souci d'homogénéité des sources, nous nous focalisons ici sur l'analyse du matériau archivistique recueilli et donc sur l'analyse des discours écrits et imprimés dans des tracts, des brochures, des journaux associatifs ou des magazines spécialisés, non sur les entretiens réalisés avec les militant-e-s.

5. Je propose la notion d'espace du militantisme LGBT pour définir un périmètre symbolique d'action au sein ou à la marge duquel s'inscrivent, dans un contexte donné, les organisations du mouvement. Voir : Prearo M., Le moment politique de l'homosexualité. Mouvements, identités et communautés en France, PUL, 2014.

6. Je tiens à remercier Catherine Achin et Magali Della Sudda pour leurs remarques et critiques sur une version antérieure de cet article. 
Association of Lesbian and Gay Pride Coordinators (IAL/GPC) en participant aux réunions tenues à New York du 16 au 20 octobre 1997, lors desquelles l'assemblée plénière conclusive décide la création du titre de "World Pride " pour cette grande manifestation romaine de l'an 2000 7. En 1997 se déroule en même temps à Paris une Europride qui remporte un grand succès de participation et qui suscite l'enthousiasme des promoteurs de la Word Pride 2000, qui vont jusqu'annoncer, lors de l'Europride 1998 de Stockholm la venue à Rome d'un million de personnes ${ }^{8}$.

Si, avec l'acronyme LGBT, il s'agit de rendre visible la réalité plurielle du mouvement, il s'agit aussi avant tout de produire un discours de mobilisation qui, par l'utilisation d'un discours inédit, parvienne à redistribuer les rapports de force inter-associatifs et à mieux représenter le vaste champ de la " communauté du mouvement social » LGBT 9. Ainsi, lors de l'Europride 1998 de Stockholm, la manifestation est définie, pour la première fois, après que la London Pride de 1996 eut inauguré cette dénomination, comme une "Gay, Lesbian, Bisexual \& Transgender

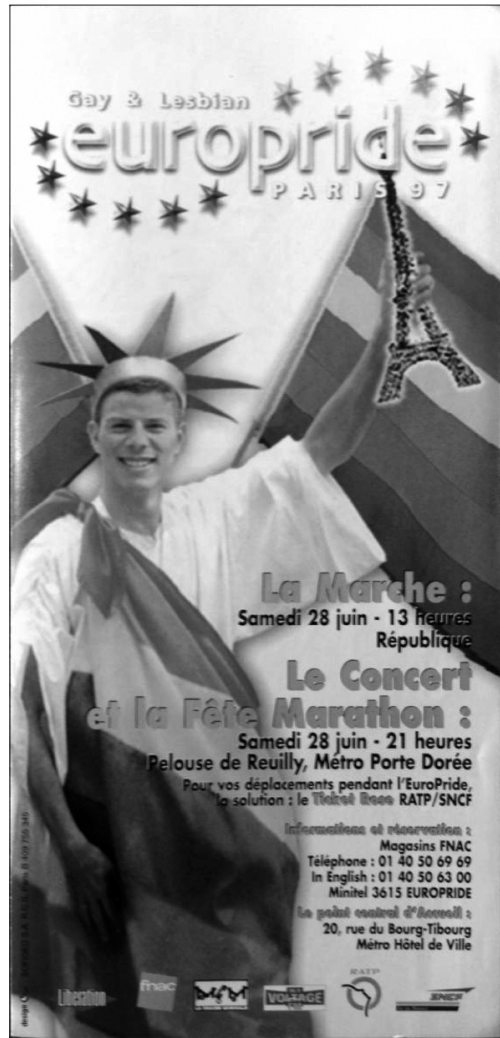

Image 1. Europride Paris 1997 Pride» :

«"L’Unité par la différence”, la manifestation européenne de la fierté gay/lesbienne/bisex/transgenre se présentera cette année avec ce slogan. » 10

7. On peut lire une reconstruction de ces événements dans un fax adressé par Renato Sabbadini, alors secrétaire démissionnaire d'Arcigay Nationale, le 3 novembre 1997 aux organes représentatifs d'Arcigay et à la Coordination pour la Pride 2000 intitulé "Questioni d'orgoglio ", Archives « Arcigay », Dossier 1997, Centro di Documentazione Omosessuale, Bologne. Le récit a été confirmé également par Imma Battaglia lors d'un entretien avec l'auteur, le 8 avril 2014. Voir également Pini A., "Roma 2000... ? ", Babilonia, 162, 1998, pp. 12-13; ainsi que Mazzotta M., "World Pride in pillole », Aut, 4/9, 1998, pp. 17-18.

8. Battaglia I., « Discorso tenuto a Stoccolma il 26 giugno 1998 », Aut, 4/9, 1998, pp. 5-6.

9. Bereni L. et Revillard A., «Un mouvement social paradigmatique ? Ce que le mouvement des femmes fait à la sociologie des mouvements sociaux ", Sociétés contemporaines, 185, 2012, pp. 17-41.

10. Lenz H., « Unity through Diversity 18-26 luglio 1998 », Aut, 4/6, 1998, pp. 18-19. 


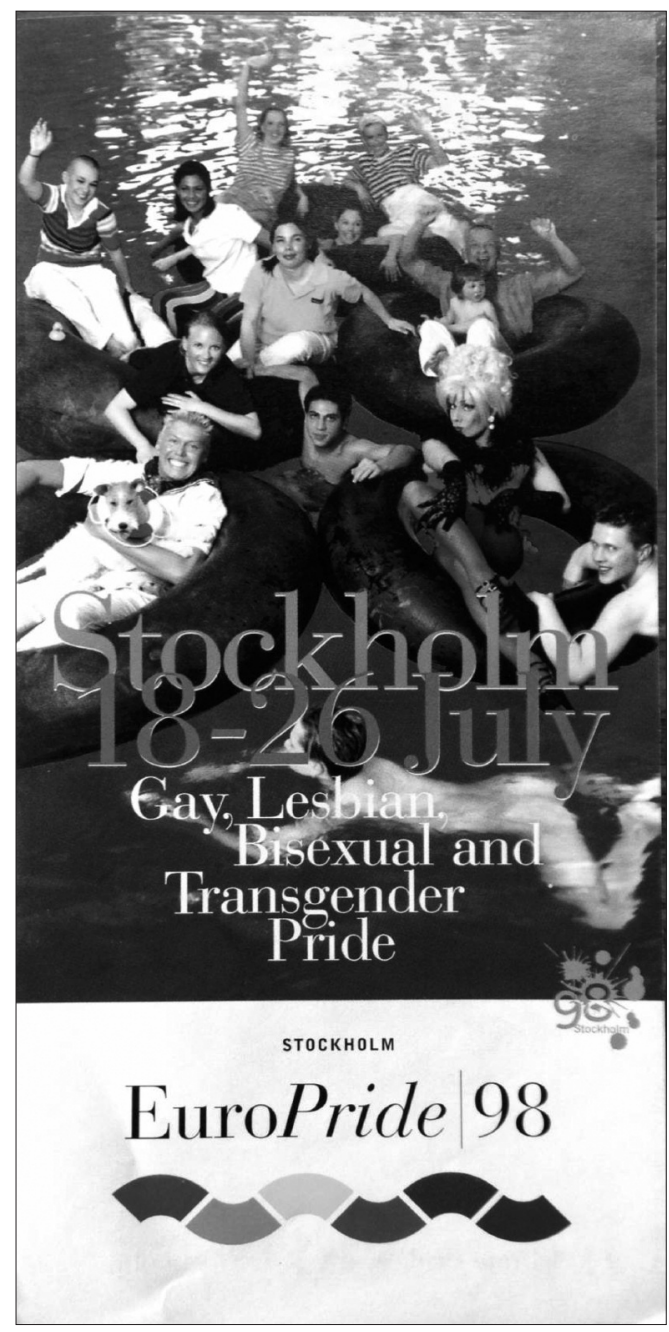

Image 2. Europride Stockholm 1998
On peut d'ailleurs observer que le visuel choisi a pour ambition précisément de refléter la pluralité des réalités qui composent le mouvement. Si la photo choisie pour la plaquette de présentation de l'Europride 1997 dépeint un jeune homme gay blanc déguisé en statue de la liberté - la référence à l'histoire états-unienne qui est à l'origine des Gay Pride avec les émeutes de Stonewall en 1969 est ici évidente -, la photo choisie pour l'Europride 1998 a pour sujet un groupes de personnes gays, lesbiennes, transexuelles, transgenres, une drag queen, de différentes couleurs de peau. L'utilisation de la formule LGBT signe ainsi la naissance d'un registre discursif militant qui pour être relié à un espace du militantisme LGBT international, n'en demeure pas moins ancré dans des contextes locaux spécifiques.

L'ambition de l'article est, au travers du regard analytique de la comparaison franco-italienne, de faire apparaître comment l'appropriation de discours de mobilisation circulant dans l'espace international des mouvements LGBT produit, au niveau local, des répertoires d'action et des stratégies militantes irréductibles à une "globalisation» des questions sexuelles 11 . J'ai donc opté pour une approche "par le bas » qui permette de montrer, à partir des contextes nationaux et de leurs différences, les usages et les appropriations des enjeux et des discours internationaux tout en soulignant la permanence de logiques locales de production du militantisme LGBT. 


\section{Les enjeux d'une comparaison par le bas}

La circulation des discours et des pratiques militants homosexuels entre la France et l'Italie, pourtant peu documentée, traverse l'histoire politique de l'homosexualité. Dès 1959, dans le colonne de la revue Arcadie, Maurizio Bellotti inaugure une rubrique « Nouvelles d'Italie » pour présenter les cultures de l'homosexualité en Italie, pour discuter de la présence des homosexuels italiens en France, mais aussi pour réfléchir sur la situation paradoxale qui voyait l'existence en France, pays des droits de l'Homme, d'une loi « antihomosexuels » ${ }^{12}$, et la situation apparemment libérale de l'Italie qui, sur fond d'un environnement fortement répressif, ne prévoyait pas la pénalisation des rapports homosexuels ${ }^{13}$. Dans le contexte de la révolution homosexuelle du début des années 1970, le Front Homosexuel d'Action Révolutionnaire (FHAR) est considéré par les militants italiens, entre autres, comme une forme d'activisme précurseur et exemplaire de la lutte révolutionnaire ${ }^{14}$. Des délégations se rendent alors régulièrement à Paris pour suivre de près les réunions des homosexuels révolutionnaires ; en Italie ce sera le FUORI (Fronte Unitario Omosessuale Rivoluzionario Italiano). Plus récemment, et notamment depuis l'adoption du PACS, la France est vue comme le pays « cousin » qui a montré la voie pour la reconnaissance des couples homosexuels. Par exemple, en 2003, l'association italienne Arcigay lance une vaste campagne de sensibilisation et de revendication intitulée «Un Pacs avanti » [Un Pacs en avant], en défendant le dispositif législatif de reconnaissance des couples non mariés comme un modèle d'inspiration pour le législateur et le gouvernement italiens. Durant la campagne électorale de 2006, le candidat de l'union de centre-gauche Romano Prodi propose, une fois élu, de faire adopter les DICO (Droits et devoirs des personnes vivant stablement ensemble), une institution juridique très proche du Pacs français, bien que cette promesse ait été abandonnée par la suite.

Enfin, ces dernières années, la France et l'Italie, contrairement à d'autres pays de l'Union Européenne, ont connu une forte contestation de ces politiques. L'émergence transnationale et transeuropéenne de contre-mouvements de défense de la famille traditionnelle et du prétendu « droit de l'enfant » à avoir un père et une mère, fervents opposants à l'ouverture du mariage

12. Il s'agit de la loi signée en 1942 par Pétain et maintenue par De Gaulle qui prévoyait une différence de traitement des rapports avec des mineurs, pénalisant davantage les rapports homosexuels ; puis du fameux amendement Mirguet qui classait l'homosexualité parmi les «fléaux sociaux ». Voir : Idier A., Les alinéas au placard. L'abrogation du délit d'homosexualité (19771982), Paris, Cartouche, 2013.

13. Pini A., Quando eravamo froci. Gli omosessuali nell'Italia di una volta, Milano, Il Saggiatore, 2011.

14. Ces échanges ont été documentés dans : Prearo M., «La trajectoire révolutionnaire du militantisme homosexuel italien dans les années 1970 », Cabiers d'bistoire. Revue d'bistoire critique, 119, 2012, pp. 79-97 ; et aussi dans Prearo M., "La "follie” radicale de Mario Mieli », in Mieli M., Éléments de critique homosexuelle. Italie : les années de plomb, Paris, EPEL, 2008, pp. 9-23. 
et de l'adoption aux couples de même sexe, constitue un nouvel élément qui invite à rapprocher analytiquement les contextes français et italien. L'organisation concomitante en France et en Italie, le 5 octobre 2014, de manifestations pour la défense de la «famille traditionnelle» de la part des groupes s'inspirant du mouvement de "La Manif pour tous », et de contre-manifestations contestataires, montre qu'on assiste à une convergence transnationale franco-italienne de l'action collective ${ }^{15}$.

Mais, au-delà de la transnationalisation des questions LGBT 16, la comparaison franco-italienne permet aussi d'étudier par contraste des situations sensiblement différentes, marquée pour la France par un contexte de reconnaissance et pour l'Italie par un contexte d'inopportunité politique, sur fond d'une même définition de la cause LGBT en termes d'égalité des droits. En effet, accompagnant et, dans certains cas, anticipant les dispositifs nationaux, l'Union Européenne a constitué, dès l'introduction de l'art. 13 du Traité d'Amsterdam (1997) instituant la compétence européenne en matière de lutte contre les discriminations fondées sur la « race ou l'origine ethnique, la religion ou les convictions, l'âge, le handicap et l'orientation sexuelle », un laboratoire d'élaboration de référentiels de l'action publique ${ }^{17}$, qui a œuvré à l'introduction du principe d'égalité dans « l'espace européen des politiques publiques ${ }^{18}$. Attachées dans un premier temps à réguler l'égalité des sexes dans le marché du travail ${ }^{19}$, les politiques européennes d'égalité se sont progressivement élargies à la suite de la proclamation de la Charte des droits fondamentaux de l'Union Européenne (2000) : d'abord avec l'adoption de la « directive relative à l'égalité raciale » (2000) 20 , ensuite avec la proposition d'un « Programme d'action contre la discrimination » (2001-2006), puis avec la Recommandation du Conseil de l'Europe CM/Rec(2010)5 du Comité des Ministres aux États membres sur « des mesures visant à combattre la discrimination fondée sur l'orientation sexuelle ou l'identité de genre », ou avec la résolution du Parlement européen (2011) sur «l'orientation sexuelle et l'identité de genre ». L'Union Européenne a ainsi défini un champ d'action et dési-

15. Simeant J., «La transnationalisation de l'action collective », in Agrikoliansky E. et al., Penser les mouvements sociaux. Conflits sociaux et contestations dans les sociétés contemporaines, Paris, La Découverte, 2010, pp. 121-144.

16. L'histoire de l'International Lesbian \& Gay Association (ILGA) est à ce propos particulièrement significative. Voir : Ayoub P., Paternotte D., "Building Europe: The ILGA and LGBT Activism in Central and Eastern Europe”, Perspectives on Europe, 42, 2012, pp. 50-56.

17. Muller P., "Référentiel », in Boussaguet L. et al., Dictionnaire des politiques publiques, Paris, Presses de Sciences Po, 2010, pp. 555-562; Muller P., «Esquisse d'une théorie du changement dans l'action publique. Structures, acteurs et cadres cognitifs ", Revue française de science politique, 55/1, 2005, pp. 155-187.

18. Muller P., "L'européanisation des politiques publiques ", Politiques et management public, 15/1, 1997, pp. 3-9.

19. Jacquot S., L'égalité au nom du marché ? Émergence et démantèlement de la politique européenne d'égalité entre les hommes et les femmes, Bruxelles, Bern, Berlin, Frankfurt am Main, New York, Oxford, Wien, Peter Lang, 2014.

20. Guiraudon V., "Construire une politique européenne de lutte contre les discriminations l'histoire de la directive "race" ", Sociétés contemporaines, 53, 2004, pp. 11-32. 
gné un nouveau domaine d'intervention 21. Ainsi, l'introduction et l'affirmation de l' "égalité des droits » comme référentiel de mobilisation est redevable tant du contexte national que de l'ancrage de la cause LGBT dans un espace symbolique de valeurs pensé et pratiqué selon une logique d'internationalisation et, surtout, d'européanisation.

Il ne s'agit pas, dans cet article, de saisir les effets d'importation d'un pays vers un autre de formes de militantisme LGBT, mais plutôt de faire apparaître par la comparaison de deux configurations politiques profondément différentes - l'une plutôt favorable aux revendications LGBT, l'autre plutôt hostile - comment une même problématisation de la question LGBT, et plus précisément l'émergence d'un mouvement qui se définit de façon inédite comme «LGBT », suit des logiques internes à l'espace national des mouvements sociaux, avant de converger au moment clé de la mobilisation internationale à l'occasion de la World Pride 2000 vers une nouvelle séquence historique de ces mouvements.

Dans une première partie, nous présenterons brièvement des éléments $\mathrm{du}$ contexte entre la fin des années 1990 et la deuxième moitié des années 2000 pour illustrer le «modèle » français de reconnaissance et le « modèle » italien de résistance aux revendications LGBT, afin de faire apparaître les différences substantielles entre les deux cas d'études. Dans une deuxième partie nous étudierons comment ces deux logiques ont produit des dynamiques inter-associatives fédératives et collaboratives en France et des dynamiques concurrentielles et conflictuelles en Italie. Plus précisément encore, il apparaîtra que si, dans le cas français, l'usage de la formule LGBT intervient, dans un certain sens « après-coup » pour consolider les acquis de la reconnaissance politique conférée par les institutions, notamment avec l'adoption du Pacs, et impulser une nouvelle phase de mobilisation, dans le cas italien, la recherche de nouveaux discours de mobilisation et donc de nouvelles stratégies militantes répond à l'exigence de restructurer, en interne, un mouvement qui paraît fortement dominé par des associations annexées par la politique de lobbying parlementaire, mais très peu incisif.

\section{Fortunes et infortunes des revendications LGBT : éléments de contexte}

\section{Politiques de la reconnaissance française}

Malgré les fortes oppositions manifestées par le mouvement de la « Manif pour tous ", l'adoption par le Parlement français de la loi n 2013-404

21. «Au regard du droit international, tous les êtres humains naissent libres et égaux en dignité et en droits. L'orientation sexuelle et l'identité de genre sont reconnues comme des motifs de discrimination interdits. [...] Par conséquent, l'Assemblée appelle les États membres à traiter ces questions ». Conseil de l'Europe, résolution 1728/2010 sur la discrimination sur la base de l'orientation sexuelle et l'identité de genre, art. 2. 
« ouvrant le mariage aux couples de personnes de même sexe » constitue une victoire certaine pour les mouvements LGBT. Depuis le « Manifeste pour l'égalité des droits » publié dans le journal Le Monde par Daniel Borrillo et Didier Eribon en mars 2004 et depuis le « mariage de Bègles », célébré en juin 2004 par le Député-Maire Noël Mamère dans sa ville ${ }^{22}$, les mouvements LGBT français ont fait de la rhétorique de l'«égalité des droits » une revendication cruciale pour la reconnaissance des droits des personnes LGBT.

L'adoption de la loi sur le «mariage pour tous » survient dans un contexte européen d'égalisation progressive 23 - malgré des résistances structurelles persistantes, comme en Italie. La loi Taubira est une des expressions d'une logique de reconnaissance qui a traversé les années 2000. En effet, on peut identifier au moins quatre temps forts de ce que l'on peut appeler une politique de la reconnaissance en France : l'adoption du Pacs en 1999, l'adoption de la loi de 2004 sur la pénalisation des propos homophobes, le discours du Président Jacques Chirac le 25 avril 2005 faisant explicitement référence à la déportation des homosexuels, et enfin, donc, la loi ouvrant accès au mariage pour les couples de même sexe en 2013.

Les théories de la reconnaissance tendent à mettre l'accent sur les revendications politiques des groupes ${ }^{24}$, mais s'intéressent moins souvent aux conséquences d'une reconnaissance acquise. En conférant une légitimité aux demandes exprimées et une efficacité à l'action militante, la reconnaissance institutionnelle attribue une valeur politique à ce qui est pensé et construit comme une entité que les acteurs en contexte définissent alors comme un « mouvement ». Après avoir introduit le cas d'étude comparatif que représente la situation italienne, on procèdera à une analyse des effets mobilisateurs produit par cette reconnaissance acquise.

\section{Une politique inopportune : le cas italien}

En Italie, malgré le fait que dans les dernières décennies plusieurs propositions de loi aient été présentées ${ }^{25}$, aucune loi reconnaissant les couples de même sexe et les droits annexes n'a jamais été adoptée. Hormis des dispositions en matière de lutte contre les discriminations au travail adoptées en

22. Pour une reconstruction de cette mobilisation et sur les étapes qui ont conduit à la célébration dissidente de ce mariage voir Eribon D., Sur cet instant fragile... Carnets janvier-août 2004, Paris, Fayard, 2004 ; ainsi que Borrillo D., « Histoire du mariage pour tous: les origines provinciales ", Médiapart, 29 janvier 2013. Consulté le 20 février 2014. URL : http://blogs.mediapart.fr/blog/daniel-borrillo/290113/histoire-du-mariage-pour-tous-lesorigines-provinciales

23. Paternotte D., Revendiquer le « mariage gay». Belgique, France, Espagne, Bruxelles, Éditions de l'Université de Bruxelles, 2011.

24. Taylor C., Multiculturalisme : différence et démocratie, Paris, Aubier, 1994.

25. Winkler M., Strazio G., L'abominevole diritto. Gay e lesbiche, gindici e legislatori, Milano, Il Saggiatore, 2011. 
$2003^{26}$, le législateur italien oppose une ferme résistance aux revendications portées par les mouvements LGBT et semble imperméable aux politiques antidiscriminatoires promues au niveau européen 27.

D’un côté, on peut souligner le cadrage que le pouvoir ecclésiastique impose au champ politique par une présence offensive particulièrement aguerrie dans le but de défendre un pouvoir temporel que la classe politique alimente, protège et promeut pour s'assurer les faveurs d'un électorat qu'elle continue - malgré les « révisions » que suggère la sociologie politique ${ }^{28}$ - de considérer comme foncièrement catholique et lié aux doctrines religieuses édictées par l'Église Catholique et par le Pape. L'hégémonie de la Démocratie Chrétienne durant plus de 30 ans, puis de la droite berlusconienne dans les vingt dernières années ont sans aucun doute, de ce point de vue, largement contribué à vitaliser l'empreinte culturelle catholique qui continue d'influencer les débats autour des «questions de société ».

De l'autre, on peut aussi observer que l'instabilité politique des majorités parlementaires, les courtes durées de vie des législatures italiennes, le recours en situation de crise politique à des gouvernements dit « techniques » qui ne sont pas issus des élections, allant jusqu'à la situation des alliances gouvernementales entre le centre-gauche et le centre-droit, ont joué en défaveur du mouvement LGBT dont l'agenda revendicatif s'est donc souvent vu renvoyer d'élection en élection et de législature en législature. L'actuel Premier Ministre Matteo Renzi a annoncé sa volonté de présenter une proposition de loi reconnaissant les unions civiles des couples homosexuels qui recouvrirait les droits liés au mariage, à l'exclusion du droit d'adopter, inspiré du modèle allemand des civil parternships. Approuvée par la commission justice de la Chambre des députés, la proposition de loi est actuellement en discussion au Sénat, où l'opposition a déposé environs 4000 amendements pour empêcher ou retarder le vote de la commission.

Cependant, si le législateur italien ne s'est jamais engagé dans l'extension du domaine de la «démocratie sexuelle » 29 , le Parlement italien a été probablement l'un parmi ceux des pays européens avec le plus fort taux de députés ouvertement gays, lesbiennes et transgenres ${ }^{30}$. Pour expliquer ce phénomène,

26. Le décret législatif $\mathrm{n}^{\circ} 216$ du 9 juillet 2003 intègre ainsi la directive européenne 2000/78/CE sur la pénalisation des mesures discriminatoires à l'embauche et dans le milieu du travail fondées sur l'orientation sexuelle.

27. Pour une analyse du rôle de l'Union Européenne dans la promotion de droits égalitaires et de dispositifs juridiques de protection des minorités sexuelles voir Borrillo D., "La politique antidiscriminatoire de l'Union européenne ", in Borrillo (ed.), Lutter contre les discriminations, Paris, La Découverte, 2003, pp. 139-152.

28. Sani G., "Il mercato elettorale rivisitato », Rivista Italiana di Scienza Politica, 3, 2006, pp. 351-362.

29. Fassin É., Le sexe politique. Genre et sexualité au miroir transatlantique, Paris, EHESS, 2009.

30. Par exemple, au cours de la XVe législature de la République italienne (2006-2008), on peut compter 4 députés et sénateurs, élus des partis de gauche et de centre-gauche, provenant du 
on peut sans doute invoquer la conjoncture favorable qui a permis à ces candidatures de se retrouver dans des positions éligibles au sein des partis de gauche et de centre-gauche à un moment où les formations s'opposant à l'hégémonie politique de Silvio Berlusconi étaient à la recherche d'une base électorale la plus vaste possible. Mais on doit également souligner à quel point cette présence LGBT au Parlement est aussi le fruit d'une stratégie politique qui a longtemps occupé le mouvement LGBT italien et plus particulièrement l'organisation Arcigay, l'association nationale qui, au fil du temps, et malgré les critiques des groupes LGBT se retrouvant dans une position dominée à l'intérieur de l'espace du militantisme LGBT italien, a joué un rôle de premier plan dans la conception et la mise en œuvre de stratégies nationales de défense et de promotion des droits LGBT ${ }^{31}$.

Arcigay a été en effet un laboratoire politique où les activistes LGBT, comme Franco Grillini, son premier Secrétaire (1985), élu en 1987 Président, puis en 1998 Président Honoraire, se sont engagés dans une carrière politique en affichant la conviction que, face aux résistances religieuses et culturelles caractéristiques du contexte historico-politique italien, un des seuls moyens pour obtenir des avancées en matière de défense et promotion des droits des minorités sexuelles était de changer de l'intérieur les dispositifs législatifs. La stratégie d'Arcigay a donc été, dès le début, une stratégie de présence et de visibilité politiques. Pourtant, dans le cas spécifique du mouvement LGBT italien et du positionnement de la classe politique italienne vis-à-vis des questions LGBT, il semblerait que l'on assiste à une politique d'inopportunité structurelle auxquelles le mouvement LGBT est amené à faire face depuis plusieurs décennies ${ }^{32}$.

monde militant LGBT : Franco Grillini (qui fut cofondateur et Président de Arcigay), Gianpaolo Silvestri (parmi les fondateurs de Arcigay) Titti de Simone (à l'époque Présidente de Arcilesbica) et Vladimir Luxuria (transgenre et activiste au sein du monde associatif LGBT à Rome). La XVIe législature (2008-2013) comptait en outre la présence d'Anna Paola Concia (activiste LGBT). À l'issue des élections, la XVIIe législature, l'actuelle, comptait, outre le député Scalfarotto, ouvertement gay mais pas réellement activiste, la présence de Sergio Lo Giudice (qui fut Président de Arcigay), de Nichi Vendola (actuellement Président de la Région des Pouilles et activiste LGBT) et de Alessandro Zan (activiste LGBT), tandis que d'autres candidatures ouvertement LGBT n'ont pas pu être élues, faute de voix ou de position de liste favorable.

31. Voir, par exemple, l'autobiographie politique de Franco Grillini, Ecce homo. 25 anni di rivoluzione gentile, Milano, Rizzoli, 2008 ; ainsi que Gianni Rossi Barilli, Il movimento gay in Italia, Milano, Feltrinelli, 1999.

32. Je reprends, en le resignifiant, le concept de structure des opportunités politiques, souvent mobilisé dans la sociologie des mouvements sociaux pour analyser la façon dont les institutions peuvent favoriser ou entraver la réussite de l'action collective d'un mouvement social : McAdam D., McCarthy JD., Zald M. (eds.), Comparative Perspectives on Social Movements, Cambridge, Cambridge University Press, 1996. Pour une discussion des modèles théoriques actuels de la sociologie des mouvements sociaux voir Fillieule O., Agrikoliansky E., Sommier I., Penser les mouvements sociaux. Conflits sociaux et contestations dans les sociétés contemporaines, Paris, La Découverte, 2010. 


\section{Discours de mobilisation et mobilisation des discours}

\section{Le devenir LGBT du mouvement bomosexuel français}

Le débat autour du Pacte civil de solidarité (Pacs) constitue, en France, un moment marqué par une dynamique inter-associative de rassemblement politique. La tristement fameuse manifestation de la « génération anti-Pacs » du 31 janvier 1999 conduit, en effet, les associations à se rassembler pour apporter une réponse unitaire, qui prendra la forme du Manifeste pour une stratégie contre l'bomophobie, rédigé à l'initiative du Centre gai et lesbien, d'AIDES, de Prochoix et de SOS homophobie :

«Ce que nous voulons, c'est une véritable stratégie répressive et préventive contre l'incitation à la haine et les discriminations. Pour en finir avec le rejet, l'intolérance et le mépris. Pour ne plus laisser les victimes de l'homophobie seules avec leur lutte. » 33

Après l'adoption par le parlement de la loi sur le Pacs, on observe la naissance de l'association Lesbian \& Gay Pride Île-de France qui a pour objectif de réunir les associations de la région parisienne pour faire de cette reconnaissance politique l'occasion d'une refondation du mouvement. L'objectif est alors d'élaborer des stratégies d'action politique commune. Réunies dans la Lesbian \& Gay Pride Île-de-France, les associations LGBT rédigent en mai 2000 un « Livre blanc » avec l'ambition de valoriser l'histoire du mouvement et de présenter une nouvelle phase de mobilisation :

«Vingt ans après le Manifeste du CUARH, neuf ans après le Livre blanc du Collectif Gay Pride, et au lendemain de l'entrée en vigueur de la loi relative au pacs, la Lesbian \& Gay Pride Île-de-France a confié à son Conseil, formé d'une trentaine d'associations nationales ou régionales, l'élaboration d'un nouveau livre blanc. » 34

Toutefois, dès les premières pages, la division du mouvement semble préoccuper les acteurs :

«Le texte garde la trace des nombreuses discussions dont il est issu, depuis l'automne 1999 : la variété des analyses reflète la pluralité des associations qui y ont contribué. Nous n’avons ainsi pas cherché à produire un consensus sur des positions revendicatives communes au détriment de l'analyse et d'une approche critique. [...] Les associations ne sauraient donc adhérer à l'intégralité du Livre blanc,

33. Collectif, Manifeste pour une stratégie contre l'homophobie [En ligne], 1999. Consulté le 20 avril 2015. URL: www.france.qrd.org/texts/discrimination/cglparis 19991117.html

34. Lesbian \& Gay Pride Île-de-France, Livre blanc 2000 [En ligne], 2000, p. 5. Consulté le 20 avril 2015. URL : http://lgp-idf.france.qrd.org/Doc/LivreBlanc2000.pdf 
chacune se réservant, selon sa sensibilité, une marge d'interprétation qui lui est propre. » 35

L'adoption du Pacs est ici interprétée comme une victoire politique qui signe la reconnaissance obtenue et la légitimation d'un mouvement qui a su se poser en interlocuteur légitime des institutions. Les militant-e-s s'engagent alors dans une stratégie de rassemblement politique pour repenser à la fois les contours de ce mouvement et l'objet des mobilisations à venir :

«Une étape décisive a été franchie avec la création du Pacs, qui dégage l'horizon de nos revendications et nous oblige à faire un nouveau travail : celui de retrouver nos marques dans le foisonnement des revendications qui se sont fait jour au fur et à mesure que les homosexuel / les ont commencé à prendre clairement conscience et à verbaliser les multiples discriminations auxquelles il / elles étaient en butte. » 36

Il s'agit d'établir une plateforme d'actions collectives communes qui trouve son point d'orgue dans l'organisation et dans la réalisation annuelles de la Marche de la Lesbian \& Gay Pride. Cette manifestation est identifiée par les associations comme un moment fondamental qui scelle, en même temps, l'unité stratégique et la pluralité des points de vue : « la méthode est celle du consensus fondé sur un pacte de respect mutuel et de volonté d'avancer ensemble afin de faire profiter nos préoccupations particulières de l'élan commun et du retentissement de la marche " ${ }^{37}$. L'association est d'ailleurs fondée sur le principe de l'action inter-associative, comme indiqué dans le Rapport d'activités 2001 :

"L'instance prépondérante est le Conseil, formé seulement de personnes morales. Son rôle est de discuter et décider des grandes orientations de l'association. C'est aussi un lieu public de rencontre et d'échange pour ses membres, et un lieu de mise en commun de moyens et d'élaboration de stratégies collectives. Le Conseil élit son porte-parole, lequel est de droit président de l'association. Deux commissions sont constituées au sein du Conseil : la commission politique et la commission inter-associative, chacune désignant ses secrétaires. La commission politique a pour objet d'élaborer une stratégie commune, de produire les documents et de participer au dialogue politique et social. La commission inter-associative a pour objet d'élaborer et de mettre en œuvre des projets communs visant à accentuer la solidarité entre les associations et leur visibilité. » 38

35. Ibid.

36. Ibid., p. 7.

37. Ibid., pp. 7-8.

38. Lesbian \& Gay Pride Île-de-France, Rapport d'activités 2001 [En ligne], 2001, je souligne. 
En mai 2002, l'association change de nom et se déclare à la préfecture de police de Paris avec une nouvelle raison sociale : Interassociative lesbienne, gaie, bi et trans (Inter-LGBT). La même année 2002, d'ailleurs, l'ancienne Gay Pride, devenue ensuite Marche de la Lesbian \& Gay Pride, devient officiellement la Marche des fiertés lesbiennes, gaies, bi et trans, introduisant ainsi l'acronyme LGBT comme nouveau discours de mobilisation et nouveau discours militant.

L'adoption du label inter-associatif «LGBT », dans ce contexte de reconnaissance politique, constitue un instrument qui vise à garantir la coexistence d'une logique d'unité politique et d'une logique de division associative. C'est un point d'arrivée d'un processus d'institution politique du mouvement homosexuel qui trouve dans sa version LGBT une issue stabilisatrice. Plus précisément, l'institution en 2002 de l'inter-associativité LGBT réussit dans une même dynamique à fonder le mouvement dans une unité symbolique et à exploiter la division associative comme ressource politique.

Ainsi, à l'aube des années 2000, les stratégies de gestion politique et de mise en forme de la division ont produit, en France, plus qu'un mouvement LGBT singulier, ce que l'on peut appeler un espace du militantisme LGBT capable d'assurer l'équilibre inter-associatif, même de manière instable et sous tension permanente. Au regard des transformations intervenues à l'occasion de l'introduction du label LGBT, on peut donc affirmer que l'acronyme LGBT a fonctionné comme un opérateur du rassemblement militant interassociatif et comme un facilitateur des dynamiques concurrentielles qui traversent l'espace des mouvements sociaux dans la définition des enjeux de la lutte. Tout autre est la situation dans le contexte italien, où l'introduction de la formule LGBT a été au contraire un amplificateur des conflits inter-associatifs.

\section{Le référentiel LGBT comme discours contestataire : une exception italienne}

Comme dans le cas français, l'organisation annuelle des manifestations de la Gay Pride a constitué pendant longtemps en Italie l'événement clé autour duquel se sont concentrées les tentatives de transformations et d'innovations du militantisme LGBT. Née comme stratégie militante visant à amplifier les effets de visibilité inhérents à ce type de manifestation, inaugurée en 1994 à Rome de la volonté de la plus grande association homosexuelle italienne Arcigay, la tenue annuelle d'une "Pride nationale » 39 a été, depuis, un des

Consulté le 20 avril 2015. URL : http://lgp-idf.france.qrd.org/Asso/ra2001.html

39. En France, dans les trente dernières années, et au fur et à mesure que le mouvement se diffusait sur le territoire, plusieurs Marches des Fiertés ont existé, organisées localement et qui mobilisent au niveau de la ville ou de la Région les associations concernées. Traditionnellement, la Marche organisée à Paris est celle qui a une participation plus importante, de la part aussi des personnalités politiques, elle a donc de fait une ampleur nationale. 
rendez-vous militants les plus importants. Plus particulièrement, le choix de la ville devant héberger la tenue de la Gay Pride d'ampleur nationale sur laquelle concentrer, chaque année, les ressources humaines, médiatiques et financières disponibles, a toujours été pour le mouvement LGBT italien une occasion de conflits ouverts et de fortes tensions. En 1997, un contentieux voit s'affronter l'association Arcigay et un nouveau collectif créé, dès décembre 1994, pour contrer la tendance hégémonique de la première, à l'initiative du Circolo di Cultura Omosessuale (CCO) Mario Mieli de Rome : le collectif Azione Omosessuale [Action Homosexuelle] qui se propose d'être

« une fédération d'organisations non gouvernementales visant la défense des droits des personnes homosexuelles [...]. Une fédération qui veut mettre au premier plan la vie et la visibilité des gays et des lesbiennes, mais sans effacer toutes ces minorités sexuelles que le mouvement homosexuel a oubliées ou a voulu oublier. » 40

En réalité, l'intention première qui animait le $\mathrm{CCO}$, membre fondateur et promoteur, était de créer une organisation qui aurait pu être une « alternative à Arcigay-Arcilesbica Nationaux » ${ }^{41}$ qui s'était imposée comme une sorte de monopole militant au niveau national avec une très forte action médiatique et un tissu local en expansion d'antennes associatives « franchisées ». C'est précisément lors de la décision d'Arcigay (devenue entretemps ArcigayArcilesbica) d'organiser en 1997 une Pride Nationale à Venise, qu'Azione Omosessuale entreprend de fonder une Coordination alternative pour l'organisation d'une autre Gay Pride Nationale à Rome, la Coordination Nationale Rainbow'97 :

"C'est une nouvelle formule, transversale, fusion de la fierté gay, lesbienne, bisexuelle et transsexuelle qui se pose comme objectif contre la culture du "ghetto" (auparavant nécessaire, aujourd'hui dépassable et en partie dépassée) - une participation réelle, concrète et visible de tous : citoyens, associations, politiques, lieux alternatifs, hommes et femmes d'église, et tous ceux qui croient aux principes élémentaires de liberté. » 42

Dans le communiqué annonçant la création d'une coordination pour la tenue de la Gay Pride nationale à Rome le 28 juin 1997, on peut lire les raisons qui ont poussé les acteurs à s'engager dans une entreprise concurrente et dissidente :

40. Azione Omosessuale, «Agir pour les homosexuels, agir ensemble ! ", Aut, 1/0, 1995, p. 8.

41. D’Agostino F. (ed.), Un anno di storia gay, lesbica, bisessuale, transessuale E transgender. Annuario GLBT 1997, Roma, Stampa Alternativa, 1998, p. 60.

42. Ibid., p. 48. 
«L'expérience de la Gay Pride de Naples en 1996 a été par nous tous considérée comme extrêmement positive du point de vue de l'accueil et de la résonance que cette manifestation a eu surtout dans le Sud de l'Italie : demeure toutefois un désaccord sur la façon dont cette série d'événements (débats, rencontres, manifestation) a été organisée. En effet, ils n'ont pas été le résultat d'une concertation, d'une confrontation et d'un débat entre toutes les composantes du mouvement; ils n'ont pas été l'occasion d'un développement pour tout le mouvement comme une Gay Pride devrait l'être en offrant un moment de dialogue, de collaboration et de connaissance collective. » 43

Derrière les divergences stratégiques quant à l'efficacité d'une certaine modalité organisationnelle, on peut observer une divergence sur les principes qui devraient régir pour les acteurs les dynamiques de coopération inter-associative à l'intérieur des frontières de l'espace militant, et plus précisément sur la forme que devrait prendre cet espace.

Structuré depuis le début des années 1980 sur la base d'un principe de verticalité associative, selon lequel l'association nationale dominante, Arcigay, agissait comme référent pour la création de nouveaux groupes ou collectifs en ouvrant des antennes locales affiliées, l'espace du militantisme homosexuel italien se définissait surtout par l'action et les discours proposés par Arcigay, et donc aussi comme un espace dominé par les homosexuels masculins, donc gays. Il existait pourtant de nombreux groupes et associations qui, au niveau local, ne s'inscrivaient pas dans l'action collective promue par Arcigay. Le CCO Mario Mieli basé à Rome, né au début des années 1980, quasiment au même moment qu'Arcigay, se posait déjà dans une optique concurrentielle, devenant en quelques années une des plus grandes associations LGBT italiennes, avec un très grand nombre d'adhérents et avec un important éventail de services offerts aux populations LGBT. L' " affaire » des Gay Pride Nationales constitue donc plus qu'un problème de différends inter-associatifs. Il s'agit plutôt d'un nœud problématique qui révèle les rapports de force historiques instaurés dans le contexte militant LGBT italien et un événement autour duquel se jouent les logiques de positionnement des différentes composantes du mouvement. D'un côté, on trouve une association nationale à vocation hégémonique, de l'autre des réalités ancrées dans le territoire local qui souhaitent conserver leur autonomie politique.

C'est donc un conflit qui est saisi comme une occasion, pour les associations se trouvant dans une position dominée, de repenser et transformer la configuration de l'espace du militantisme homosexuel de l'époque :

43. Azione Omosessuale, «Per un coordinamento per il Gay-Lesbian-Transexual-Bisexual Pride », non daté. Archives «Arcigay », Dossier 1996, Centro di Documentazione Omosessuale, Bologne. 
«Cette coordination doit offrir et représenter un réel espace démocratique de débat et doit être en accès libre pour toutes les réalités du mouvement homosexuel italien. Pour que la Gay Pride devienne réellement une manifestation de tous les gays, les lesbiennes, les transsexuels, les travestis et les bisexuels, il est fondamental que la coordination [soit] un lieu de débat et de confrontation. » 44

En réponse à cette poussée contestataire lancée par Azione Omosessuale autour d'un projet de Gay Pride dissidente, les responsables d'Arcigay fournissent une analyse qui montre bien que l'organisation de la manifestation cache en réalité une lutte qui implique le bouleversement des rapports de pouvoir internes à l'espace du militantisme homosexuel :

«Depuis longtemps, dans le monde gay italien, une lutte de pouvoir pour l'hégémonie est en cours. D’aucuns seraient fatigués du rôle qu'Arcigay joue et voudraient remplacer Arcigay avec une organisation plus représentative. ${ }^{45}$

Dans ce contexte conflictuel, qui voit s'affronter deux organisations concurrentes, le recours à différents discours de mobilisation produit une reformulation du référentiel militant qui préside à l'organisation de l'espace du militantisme qui se définissait jusque lors comme majoritairement « gay ». L'introduction de la part des activistes d'Azione Omosessuale d'un référentiel extensif « gay-lesbian-transexual-bisexual » (GLBT) constitue ainsi ce que Michel Foucault appelle un "événement discursif » ${ }^{46}$. Comme l'écrit Imma Battaglia, alors Présidente du CCO Mario Mieli, à l'occasion du projet de « Pride 1998 », il ne s’agit pas seulement de s'engager dans des choix stratégiques divergents, mais bien de proposer une nouvelle configuration militante fondée sur un principe d'horizontalité démocratique qui vise à inclure dans le processus décisionnel et dans le débat militant, toutes les réalités qui composent le mouvement, et notamment des composantes lesbiennes, bisexuelles et transgenres :

«En suivant les pas des événements de l'année dernière, une Coordination Pride Nationale a été créée dans laquelle convergent la grande majorité des réalités associatives $\mathrm{G} / \mathrm{L} / \mathrm{B} / \mathrm{T}$ italiennes. [...] L'objectif était de faire en sorte que le comité politique de la Pride, la Coordination, puisse assurer une présence plus vaste et plus représentative de tous les activistes du mouvement $\mathrm{G} / \mathrm{L} / \mathrm{B} / \mathrm{T}$, abs-

44. Azione Omosessuale, «Per un coordinamento per il Gay-Lesbian-Transexual-Bisexual Pride ", op. cit.

45. Grillini F., Barba D., « A proposito del Gay Pride », mail envoyé le 26 mars 1997 à la mailing list "Queer-it ", Archives "Arcigay ", Dossier 1997, Centro di Documentazione Omosessuale, Bologne.

46. Foucault M., "Sur l'archéologie des sciences. Réponse au Cercle d'épistémologie » (1968), Dits et écrits I, 1954-1975, Paris, Gallimard, 2001, p. 729. 
traction faite des chapelles d'appartenance. [...] Les soi-disant leaders historiques du mouvement Gay (et je souligne Gay et non $G / L / B / T)$ ont dû prendre en compte une nouvelle et pétillante réalité $\mathrm{G} / \mathrm{L} / \mathrm{B} / \mathrm{T}$ qui a su jouer un rôle de leader et qui, sans doute, n'accepte plus d'être considérée comme une vulgaire main-d'œuvre ou simplement comme une pépinière bonne à récolter des membres payants. » 47

L'usage du référentiel militant GLBT (devenu par la suite LGBT) représente ainsi un discours contestataire qui a pour objectif la (re)mobilisation de composantes du mouvement marginales ou marginalisées. Opposé au référentiel « gay » masculin, le référentiel LGBT, qui synthétise à la fois l'unité et la pluralité militantes, constitue une opération de reconfiguration d'un espace du militantisme qui sera défini à partir de ce moment comme LGBT.

\section{L'action collective LGBT au prisme des stratégies discursives}

Les travaux sur les mobilisations de lutte contre le VIH/sida 48 ont permis de mettre au jour l'existence d'un champ du militantisme homosexuel en constante restructuration et recomposition. On observe également des effets de transnationalisation des revendications et de la mobilisation ${ }^{49}$ qui produisent en retour, dans les contextes nationaux, des reconfigurations des rapports inter-associatifs, et donc plus globalement de l'espace des mouvements LGBT. Or, peu de travaux se sont concentrés sur les changements produits sur la mobilisation homosexuelle à partir d'une analyse des discours de mobilisation et plus particulièrement de la formule «LGBT» comme référentiel militant ${ }^{50}$. Par ailleurs l'histoire politique du militantisme homosexuel français et italien est marquée par des éléments de continuité et de discontinuité que peu de travaux ont explorés ${ }^{51}$. La construction sociale, l'actualisation his-

47. Battaglia I., « Pride 1998 : uniti verso il World Pride 2000 », Aut, 4/6, 1998, p. 6.

48. Duyvendak J.W., Fillieule O., " Gay and Lesbian Activism in France. Between Integration and Community-Oriented Movement ", in Barry A. et al. (eds.), The Global Emergence of Gay and Lesbian Politics. National Imprints of a Worldwide Movement, Temple University Press, Philadelphia, p. 184-213, 1999 ; Pinell P. (ed.), Une épidémie politique. La lutte contre le sida en France (1981-1996), Paris, PUF, 2002 ; Broqua C., Agir pour ne pas mourir! Les bomosexuels et le sida, Paris, Presses de Sciences Po, 2006.

49. Ayoub P., "Cooperative Transnationalism in Contemporary Europe: Europeanization and Political Opportunities for LGBT Mobilization in the European Union”, European Political Science Review, 5/2, 2013, pp. 279-310.

50. Pour une analyse détaillée de quelques référentiels militants LGBT, voir : Prearo M., "L'espace du militantisme LGBT à l'épreuve des présidentielles », Genre, sexualité É société [En ligne], Hors-série $n^{\circ} 2$ |2013, mis en ligne le 10 avril 2013, consulté le 14 mai 2014. URL : http://gss.revues.org/2701; DOI : 10.4000/gss.2701.

51. Pour le cas français : Martel F., Le rose et le noir. Les homosexuels en France depuis 1968, Paris, Seuil, 2008, a été pendant longtemps la seule référence en la matière, à côté d'autres ouvrages davantage analytiques focalisés sur des groupes militants ou des associations spécifiques. On pense notamment à Jackson J., Arcadie. La vie homosexuelle en France, de l'aprèsguerre à la dépénalisation, Paris, Autrement, 2009 et à Broqua C., Agir pour ne pas mourir! Les homosexuels et le sida, Paris, Presses de Sciences Po, 2006. Pour le cas italien, aucune 
torique et la circulation politique de l'homosexualité résultent de rapports mouvants et complexes travaillés par des dynamiques de signification discursive produites par des acteurs individuels ou des groupes dans des contextes spécifiques. S’il est donc une spécificité du militantisme homosexuel, c'est bien une pratique discursive d'énonciation de l'homosexualité comme discours politique, au-delà et contre les représentations sociales de l'homosexualité, qu'elles soient d'origine médicale 52 ou simplement le résultat d'un savoir de sens commun qui tend à la dépeindre comme une « déviance » 53.

En effet, dans la deuxième moitié du Xx $x^{\mathrm{e}}$ siècle, l'émergence et les transformations de la sémantique militante de l'homosexualité montrent comment la pratique discursive a contribué à engendrer différentes formes de militantisme et différents répertoires de mobilisation. Les premières tentatives de constitution d'organisations faites par et pour les homosexuel-le-s, dans les années 1950 et 1960, ont introduit une sémantique de l' « homophilie » - vocable qui entend insister sur la dimension affective et pas seulement sexuelle, caractérisant davantage l'expérience masculine, mais étant utilisée également de façon générale et abstraite aussi pour les lesbiennes. Elles s'inscrivent dans des contextes fortement répressifs et proposent une mobilisation plus culturelle que politique. Dans le sillage des « années 1968 » 54 , la conversion à la thématique de la révolution s'accompagne d'une révolution discursive qui pose l'homosexualité, et non plus l'homophilie, comme une forme de sexualité minoritaire dans le but de proposer une politique de la sexualité à même de défaire les structures hétéronormées de la société. Enfin, l'émergence de la sémantique «gay » entre la fin des années 1970 et le début des années 1980, signe l'avènement d'un militantisme identitaire et communautaire qui portera à la création de structures collectives sous formes d'associations avec des raisons sociales très diverses : culturelles, juridiques, politiques, sportives, confessionnelles, d'entraide, professionnelles, etc.

Durant la deuxième moitié des années 1990, l'acronyme LGBT s'est en effet peu à peu mondialement diffusé pour définir en même temps l'unité politique des communautés lesbiennes, gaies, bi et trans, et l'affirmation des spécificités identitaires; ce que l'usage pluriel du terme «homosexualités », par exemple, ou l'usage restrictif du binôme «gais et lesbiens » ${ }^{55}$, ne permettaient pas ou plus d'exprimer. L'invention de la formule «LGBT » s'inscrit ainsi dans une logique d'extension de l'espace du militantisme homosexuel et dans

étude exhaustive n'existe qui retrace l'histoire politique de l'homosexualité, hormis les quelques références citées dans cet article.

52. Foucault M., Histoire de la sexualité 1. La volonté de savoir, Paris Gallimard, 1976.

53. Borrillo D., L'homophobie, Paris, Presses Universitaire de France, 2001.

54. Zancarini-Fournel M., "Genre et politique : les années 1968 », Vingtième siècle, 75, 2002, pp. 133-143.

55. de Lauretis T., «Théorie queer : sexualités lesbiennes et gaies. Une introduction » (1991), in Théorie queer et cultures populaires. De Foucault à Cronenberg, Paris, La Dispute, 2007, pp. 95-122. 
une logique d'intégration démocratique des composantes qui se situent dans cet espace symbolique d'action collective. Au cours des années 2000, on constate un renouveau des raisons sociales des organisations qui ont toutes (ou quasiment toutes) introduit la dénomination LGBT comme une nouvelle identité associative et comme principe d'inclusion. L'hypothèse ici avancée est que bien loin de se limiter à une transformation formelle, l'adoption de la formule LGBT représente une refonte de la mobilisation.

Les deux cas d'étude présentés montrent que la définition d'une cause commune portée de concert par les associations LGBT réunies dans des organisations fédératrices, en France, ou faisant l'objet d'une forte tension et concurrence inter-associative, en Italie, est sans doute marquée par les logiques d'internationalisation pensées et pratiquées en vue de l'organisation de la première Marche des Fiertés mondiale, ayant contribué à tracer, en retour, les contours d'une cause LGBT internationalisée, mais n'en reste pas moins redevable des spécificités nationales et notamment de la structuration du champ politique et militant local. En tant que référentiel militant, la formule LGBT, apparue entre la fin des années 1990 et le début des années 2000 et devenue depuis un label mondialement reconnu et utilisé, représente un événement discursif qui a permis la reconfiguration de cet espace et la redistribution des rapports de force le structurant, selon des dynamiques de mobilisation locales spécifiques et singulières. Une analyse en termes de stratégies discursives se révèle dès lors particulièrement heuristique pour saisir l'articulation des échelles de mobilisation nationale, européenne et internationale dans la structuration de l'action collective LGBT. 